anthropology \& materialism

\section{Anthropology \& Materialism}

A Journal of Social Research

$2 \mid 2014$

The Persistence of Myth

\title{
Le cercle mythique : Walter Benjamin sur la politique et son interruption
}

The Mythic Circle. Walter Benjamin on Politics and its Interruption

\section{Aukje van Rooden}

\section{(2) OpenEdition}

\section{Journals}

\section{Édition électronique}

URL : http://journals.openedition.org/am/423

DOI : $10.4000 / a m .423$

ISSN : 2364-0480

Éditeur :

CETCOPRA, CRASSH - Center for Research in the Arts Social Sciences and Humanities, Fakultät Gestaltung - Universität der Künste Berlin

Référence électronique

Aukje van Rooden, « Le cercle mythique: Walter Benjamin sur la politique et son interruption »,

Anthropology \& Materialism [En ligne], 2 | 2014, mis en ligne le 15 avril 2014, consulté le 30 avril 2019.

URL : http://journals.openedition.org/am/423 ; DOI : 10.4000/am.423

Ce document a été généré automatiquement le 30 avril 2019.

Tous droits réservés 


\title{
Le cercle mythique : Walter Benjamin sur la politique et son interruption
}

\author{
The Mythic Circle. Walter Benjamin on Politics and its Interruption
}

\author{
Aukje van Rooden
}

\section{Introduction}

1 Il y a quelques années, une affaire remarquable secoua la politique néerlandaise. Dans un entretien de 2006 diffusé en direct par la télévision nationale, la parlementaire néerlandaise d'origine somalienne Ayaan Hirsi Ali déclara avoir menti sur son nom et son pays d'origine pour obtenir un statut de réfugiée politique lors de son arrivée en Hollande ${ }^{1}$. Même s'il s'agissait d'un secret de Polichinelle qu'elle avait déjà révélé à plusieurs reprises dans des médias moins grand public, des collègues membres du Parlement menacèrent aussitôt de lui retirer la nationalité hollandaise. Même rétrospectivement, un tel mensonge constituait pour certains députés un crime contre l'État dont elle était membre et représentante élue. Ce jugement n'était pas motivé par des raisons morales, car il n'a pas empêché les uns et les autres de manifester leur compassion pour la difficile situation des réfugiés au moment de leur arrivée. Il ne l'était pas non plus par des raisons pratiques, car renvoyer un parlementaire n'est pas sans conséquences. Il s'agissait bien pour le gouvernement hollandais de signifier par là une atteinte à son propre droit. Hirsi Ali démissionna de ses fonctions et partit pour les États-Unis, non seulement en réaction à cette polémique, mais aussi suite à une menace de mort plus que sérieuse ${ }^{2}$. Doit-on dire, dès lors, que la réaction disproportionnée de certains députés s'explique par le fait que l'ordre politique ne supporte pas le moindre soupçon de mensonge?

Une affaire plus récente et peut-être encore plus significative de la politique néerlandaise semble confirmer cette hypothèse. En 2008, le parlementaire hollandais du parti Vert, Wijnand Duyvendak, avouait, dans un livre autobiographique sur son passé d'activiste durant les années 80, avoir cambriolé le bâtiment du Ministère de l'Économie et y avoir 
dérobé des documents révélant des plans secrets pour la construction de centrales nucléaires. À l'époque, cette action anonyme fut louée par la population comme le démantèlement justifié des plans d'un gouvernement mensonger. Mais une fois que l'on apprit que l'activiste faisait désormais partie du gouvernement, cette action fut perçue comme impardonnable et entraîna la démission du parlementaire. Le fait que l'aveu de Duyvendak était surtout un signe de sa sincérité n'a en rien permis de dissiper le soupçon de malhonnêteté qu'il a fait planer sur la politique ${ }^{3}$. Ces exemples montrent qu'il y a dans la politique une exigence presque obsessionnelle de véracité et de sincérité, et ce d'autant plus dans le cas des ordres politiques modernes, qui ne se fondent plus sur une autorité irrécusable - comme celle de Dieu ou des ancêtres - garantissant leur vérité. Autrement dit : quand un ordre ne peut plus s'appuyer sur des fondements incontestables, la véracité devient plus plausible que la vérité. Comme nous le verrons, on pourrait dire, avec Walter Benjamin, que cette caractéristique remarquable de l'ordre politique moderne résulte du fait que, pour être politique, il doit se fonder, nécessairement, sur un « cercle mythique ».

\section{Comme un baron de Münchhausen / ou la difficulté de l'acte constituant}

3 Au-delà de leur contexte particulier, ces affaires politiques néerlandaises sont un signe des temps, et des temps changeants. Elles sont le signe que nous nous trouvons, non seulement aux Pays-Bas, mais dans le monde occidental et plus particulièrement en Europe, dans une période de profonde réflexion sur ce qu'est, au fond, un ordre politique. Car savons-nous encore avec certitude sur quelle base un ordre politique doit être érigé, qui peut y prendre part et pourquoi, et s'il est nécessaire d'avoir une identité commune pour qu'un ordre se forme ? Sur ces questions, nous nous trouvons, avec l'Europe du XXI ${ }^{\mathrm{e}}$ siècle, dans un temps particulièrement intéressant. À la fois évidence quotidienne et objet de discussions enflammées, l'ordre politique européen - le « projet Europe » - est un work in progress dont nous-mêmes sommes à la fois l'enjeu et les constructeurs.

4 Si bien des difficultés accompagnant la constitution d'un ordre politique remontent à la surface dans le cas de l'Union Européenne, celles-ci, cependant, sont inextricablement liées à l'acte constituant comme tel. Telle est du moins la thèse soutenue par Jean-Luc Nancy dans un article, publié dans le journal Le Monde du 29 juillet 2005 : «L'impossible acte constituant » (Nancy, 2005). Selon lui, les problèmes insurmontables posés par la constitution d'un ordre politique dans le cas de la communauté européenne sont les problèmes des communautés contemporaines en tant que telles. Les ordres contemporains, en effet, se distinguent des ordres pré-modernes en ce qu'ils ne se présentent plus comme le telos d'une cosmologie ou d'un ordre divin. L'homme contemporain, comme le dit aussi l'historien Mircea Eliade, sait et se veut créateur d'histoire. Le mythe d'origine ne suffit plus pour constituer son ordre communautaire, lequel ne peut plus passer par la transmission linéaire et non critique d'un contenu narratif de génération en génération (Eliade, 1989, p. 158). L'acte constituant doit donc plutôt être conçu comme un acte créateur, comme une création ou une invention de ce qui n'est pas encore donné. Ainsi, observe Nancy dans Le Monde, le peuple européen n'est pas un peuple donné, mais « un peuple en train de se constituer, en train de s'inventer en inventant, précisément son 'idée' ou sa 'forme'« . 
5 La difficulté d'une telle invention, qui est, bien naturellement, en même temps son aspect le plus intéressant, est qu'elle doit s'ériger pour ainsi dire dans le vide, dans le tumulte chaotique d'un entre-temps où elle n'a pas encore de fond parce qu'elle s'érige elle-même comme le baron de Münchausen qui s'est surpassé en s'extirpant lui-même du marais. Dans nos ordres politiques contemporains, l'acte constituant revient donc toujours à une auto-constitution parce qu'il n'y a pas d'autorité externe, transcendante, qui donne l'autorisation. Mais le fait qu'une communauté moderne - nous donc - invente sa propre idée et sa propre forme implique qu'il ne s'agit pas d'une idée ou d'une forme déjà prescrite, pour ainsi dire, dans un hymne primordial. C'est pourquoi le «nous » qui devrait représenter le peuple d'un ordre politique n'est pas un groupe préexistant, mais se définit seulement avec les articles du traité, avec les articles qui définissent, sur le plan le plus fondamental, par où passent les frontières qui séparent ceux qui en font partie de ceux qui n'y appartiennent pas.

6 En l'absence d'hymne primordial - ou mythe d'origine -, une telle définition a pourtant toujours quelque chose de contingent; elle est le résultat d'une décision prise dans des circonstances spécifiques, décision qui aurait pu être prise autrement et qui reste donc par principe toujours ouverte au débat. Ce constat de la contingence radicale du fondement de nos ordres m'incite à aborder la question de la communauté à travers une pensée politique dite post-fondationaliste, selon l'expression d'oliver Marchart (Marchart, 2007). La pensée post-fondationaliste met en doute l'idée d'un fondement immuable servant de socle à la communauté. Non qu'elle promeuve l'absence totale de fondement (ce qui reviendrait à un "anti-fondationalisme ») : elle rejette uniquement le caractère immuable du fondement, et affirme, dès lors, qu'il peut être remis en question, comme le démontrent les référendums sur le traité constitutionnel européen. La possibilité de remettre en question l'idée et la forme de la communauté est le corrélat de la contingence de son fondement : puisque la communauté n'est pas instituée d'un coup et une fois pour toutes, elle doit être répétée voire ré-affirmée à chaque fois. Une communauté qui n'a pas d'origine unique mais doit rejouer sans cesse son origine, est donc, par principe, un work in progress. Dans les ordres politiques contemporains, une telle remise en question perpétuelle et fondamentale est néanmoins impossible selon Benjamin. Bien qu'ils ne puissent pas se fonder sur une autorité irrécusable, ces ordres veulent se présenter non pas comme une œuvre ouverte au débat, mais comme bien achevés et consolidés. Une telle consolidation est trouvée dans le mythe. Autrement dit, n'ayant pas d'origine unique ou transcendante, l'ordre politique moderne a besoin d'un récit d'origine pour se savoir créateur d'histoire.

\section{Politique et mythe / ou notre politique est-elle mythique?}

7 Un des buts de cette contribution est donc de considérer, à l'exemple de Benjamin, la réflexion actuelle sur l'ordre politique en terme de mythe. Cela ne va pas de soi. $\mathrm{Ne}$ venons-nous pas de constater que ce sont les ordres pré-modernes qui sont à base mythique, alors que nos ordres se caractérisent au contraire par le fait qu'ils rejettent tout recours à un mythe d'origine? C'est certes exact. Cependant, il est possible de soutenir que les ordres politiques dans lequels nous vivons actuellement font appel à une certaine structure mythique. Cette perspective contrariante nous a été livrée par Benjamin dans un essai important qui non seulement a mis sens dessus dessous la 
conception habituelle de nos ordres politiques contemporains, mais qui, ce faisant, a également étalé au grand jour les dessous de la construction de ces ordres. Je parle de l'essai «Zur Kritik der Gewalt» [ «Critique de la violence»] ${ }^{4}$. Ce texte balbutiant et brûlant de hardiesse juvénile a beau manquer, ici et là, de solidité et de clarté conceptuelle, il nous livre un diagnostic sur nos systèmes politiques contemporains dont la force explicative est, à mon avis, loin d'être épuisée.

En quoi consiste donc la perspective révolutionnaire de ce texte "notoirement difficile » (Butler, 2006, p. 202) et «terriblement équivoque» (Derrida, 2005, p. 67) ${ }^{5}$ de Benjamin ? Elle consiste, pour la résumer un peu librement, dans le fait que les ordres politiques contemporains dissimulent, volens nolens, la contingence de leur origine et que cette dissimulation prend la forme d'une structure mythique. Pour saisir la portée de cette thèse, il faut noter qu'elle est motivée par la constatation que l'origine d'un ordre politique est non seulement contingente, comme nous l'avons déjà constaté, mais qu'elle est pour cette raison aussi et précisément, violente. Parce que l'ordonnance politique proposée par la constitution aurait pu être différente, elle implique nécessairement une décision forcée. Benjamin souligne que la violence de la décision originaire n'est pas le mal nécessaire propre à un moment qui pourrait être dépassé, mais, bien au contraire, que cette violence continue à se montrer dans la réalité quotidienne de l'ordre politique. L'exécution de la législation, par exemple, n'est jamais une simple application des lois données, mais demande, pour chaque cas spécifique, une nouvelle décision sur l'ordonnance de l'ordre. On pourrait donc dire que la «Critique de la violence » offerte par Benjamin est surtout une critique de la violence cachée impliquée par un ordre politique. Par «violence», il ne faut pas forcément comprendre la force brute des actes d'oppression : le mot allemand Gewalt signifie non seulement « force brute », mais aussi, et tout simplement, « autorité », « gouvernement » (walten = régner, gouverner).

9 Le mot allemand illustre parfaitement l'ambiguïté qui caractérise la constitution des ordres politiques contemporains. En effet, si la constitution instaurant le pouvoir autorisé ne peut s'autoriser d'aucune légitimité antérieure, c'est parce que le moment de constitution se produit dans un espace "vide», qui n'est pas encore structuré par l'opposition du légal et de l'illégal. L'enjeu de l'essai de Benjamin est d'attirer l'attention sur ce vide légal et non sur la structure du pouvoir gouvernemental en tant que tel. Benjamin précise que la violence d'un ordre politique se compose de deux dimensions : la violence fondatrice (rechtsetzende Gewalt) et la violence conservatrice (rechtserhaltende Gewalt ). Selon lui - et ceci est un des points cruciaux de son diagnostic -, ces dimensions de fondation et de conservation ne sont pas isolées et successives; elles sont inextricablement imbriquées l'une dans l'autre, parce que la fondation de l'ordre anticipe toujours sur sa conservation et que, inversement, la conservation implique toujours un moment fondateur ${ }^{6}$. Or, suggère Benjamin, l'enchevêtrement cyclique de fondation et de conservation est un enchevêtrement mythique. La violence impliquée dans le fonctionnement de l'ordre politique est donc de la violence mythique (Benjamin, 1977, p. 197sq).

10 Pourquoi qualifier le fonctionnement de l'ordre politique contemporain de «mythique »? D’abord pour souligner la continuité qui existe entre les sociétés prémodernes mythiques et notre monde contemporain. Cette continuité réside dans un certain rapport entre droit et violence, un rapport dont la structure - à la fois temporelle et narrative - s'explique en terme de destin [Schicksal] ${ }^{7}$. De même qu'autrefois les dieux grecs imposaient du droit par leur intervention soudaine, l'ordre de droit contemporain 
se caractérise selon Benjamin par une "schiksalmäßiger Gewalt [violence s'imposant à la manière d'un destin]» (Benjamin, 1977, p. 196). Comme l'ordonnance des dieux grecs, l'ordonnance de l'ordre de droit a beau être en un sens contingente, elle est, selon Benjamin, «im Sinne des Rechts nicht Zufall [accident], sondern Schicksal [destin]» (Benjamin, 1977, p. 199). Dans son texte 'Schicksal und Character' il souligne également que le destin ne présente pas l'ordre politique comme contingent, mais comme une vie qui est « condamnée » à être ainsi (Benjamin, 1977, p. 175). Ce destin, qui couronne la violence de l'ordre contemporain (Benjamin, 1977, p. 188), doit donc donner l'impression que cet ordre - son "idée » et sa "forme » - a été prédestiné par l'histoire, que sa constitution était écrite dans la chair des choses, bref, que l'ordre ne peut être que tel qu'il est. On pourrait dire que ce destin transforme le déroulement de l'histoire d'un récit ouvert en une intrigue - ainsi que le muthos aristotélicien fut traduit -, c'est-à-dire en une succession prédestinée d'événements 8 .

11 Bien que l'homme contemporain se reconnaisse et se veuille historique, il s'empêche néanmoins, par ce recours au concept de destin, d'assumer un ordre construit par l'effet d'une décision contestable - sous peine de sombrer dans le relativisme ou le nihilisme. $\mathrm{Si}$ dans la pratique quotidienne, suggère Benjamin, ce recours ne saute pas aux yeux, c'est parce qu'au lieu de se présenter dans la terminologie théologique du « destin », il revêt la forme généralement acceptée de la rationalité instrumentale. Les ordres contemporains dissipent le sentiment inconfortable de contingence en déterminant une certaine fin spécifique et en comprenant toutes les mesures prises comme des moyens d'atteindre cette fin. Aujourd'hui, l'illustration la plus claire de ce dogme est sans doute la rhétorique propre à des slogans comme "clean weapons " et "preventive war ", où les adjectifs indiquent que la violence exécutée par l'État relève d'une mesure nécessaire - et donc « innocente »- afin d'atteindre la fin qu'il s'est proposé ${ }^{9}$. Bien qu'on puisse avancer que le recours à un tel raisonnement mythique est inévitable si l'on veut faire de la politique, le point délicat réside dans le fait que la distinction entre, d'une part les fins justes et les moyens justifiés et, d'autre part les fins injustes et les moyens injustifiés (in casu, entre une "guerre préventive » et une guerre inutilement sanglante) n'est concevable qu'à partir de l'ordre constitué (Benjamin, 1977, p. 181). L'imbrication des moyens et des fins est donc une façade derrière laquelle s'abrite l'absence de justification fondamentale des fins visées.

12 Parce que son efficacité dépend de cette façade, le souci premier de l'ordre politique n'est pas, comme nous sommes enclins à le penser, celui de réaliser les fins justes qu'il a déterminées, mais de maintenir son droit exclusif à déterminer la frontière entre la violence sanctionnée et celle qui ne l'est pas, c'est-à-dire à se maintenir soi-même (Benjamin, 1977, p. 183). Néanmoins, ce souci détourne l'attention de ce que Benjamin nomme le «caractère lourdement problématique » de tout ordre de droit, à savoir « die seltsame und zunächst entmutigende Erfahrung von der letztlichen Unentscheidbarkeit [ le caractère finalement indécidable] aller Rechtsprobleme " (Benjamin, 1977, p. 196). Parce que l'ordre proposé ne peut s'étayer sur un fondement immuable qui en garantit la justesse, toute décision prise sur la base de cette ordonnance est inévitablement provisoire. Malgré la volonté d'ouverture continuelle au débat affichée par le régime démocratique, Benjamin estime qu'il est incapable de questionner la justesse de son discours de légitimation, et ce en raison de son souci premier : se maintenir soi-même (Benjamin, 1977, p. 180). Le work in progress que serait la communauté ne peut donc progresser que selon des voies déjà tracées. 


\section{Le langage du mythe / ou le règne de la représentation}

13 C'est sur ce point que la temporalité du mythe se mêle à sa structure langagière. Car, comme je l'ai déjà souligné, un ordre politique qui se forme lui-même invente non seulement ses propres normes et formes, mais les explique généralement en les enveloppant dans un récit : un récit d'origine donc. C'est, bien entendu, par un tel récit des origines que la notion de destin s'est répandue. Comment le mythe se récite-t-il alors? Plus que Benjamin lui-même, c'est Jacques Derrida qui a dégagé cette structure langagière dans son analyse du texte de Benjamin. Dans Force de loi, Derrida avance que la violence qui fonde un ordre politique répond à la catégorie grammaticale du futur antérieur :

«Comme ce droit à venir [du nouvel ordre à fonder, AvR] légitimera en retour, rétrospectivement, la violence qui peut heurter le sentiment de justice, son futur antérieur la justifie déjà [...]. Dans ces situations dites fondatrices du droit ou d'État, la catégorie grammaticale $d u$ futur antérieur ressemble encore trop à une modification du présent pour décrire la violence en cours. Elle consiste justement à feindre la présence ou la simple modalisation de la présence. Ceux qui disent 'notre temps', en pensant alors 'notre présent' à la lumière d'une présence future antérieure ne savent pas très bien, par définition, ce qu'ils disent » (Derrida, 2005, p. 87/8).

14 Ainsi qu'on l'a vu, la constitution d'un ordre a nécessairement lieu dans un « vide » où il n'y a pas encore de droit qui pourrait en être la justification. Or, comme il n'est pas souhaitable pour un ordre politique de crier sur tous les toits l'absence d'un fondement ultime le justifiant, les détenteurs du pouvoir recouvrent ce manque à la fois par une projection de ce qui est à venir et par une légitimation après coup de ce qui s'est passé. Autrement dit, recourir au «futur antérieur " permet d'enjamber ce vide en présentant un ordre de droit qui aura été ainsi ${ }^{10}$.

Voici donc décrit le geste à la fois paradoxal et inévitable de l'acte constituant. Car, comme nous le voyons aujourd'hui par exemple dans nos tentatives pour établir un ordre européen, un ordre politique ne peut qu'anticiper sur les faits afin de s'établir en tant que tel : on est forcé, en d'autres termes, de parler au nom d'un «nous » qui, à strictement parler, n'existe qu'après avoir été prononcé. De cette façon, la structure linguistique du futur antérieur dissimule l'historicité du présent en pliant le temps sur lui-même et, parallèlement, révèle l'indécidabilité de l'histoire. Comme Derrida l'a suggéré ailleurs à plusieurs reprises, il s'agit ici de deux faces de la même médaille: le futur antérieur évoque une structure temporelle qui à la fois détermine et révèle qu'il n'y a rien à déterminer ${ }^{11}$. Dans ce cadre, l'acte langagier de la promesse est exemplaire : il se donne comme une garantie là où on sait pertinemment que rien n'est garanti. Si le cours de l'histoire était garanti, il ne serait nullement nécessaire de promettre. Et en fin de compte, faire de la politique, est-ce autre chose que faire des promesses? Alors que Derrida, parmi d'autres, a surtout insisté sur le fait que la forme grammaticale du futur antérieur ouvre, dans le présent, la possibilité d'un à-venir qui fait place au tout autre, il ne faut pas, à mon avis, sous-estimer la suggestion de Benjamin : le futur antérieur est aussi la force motrice de la politique mythique. pour objectif d'abolir le cours de l'histoire, de créer un ordre trans-historique, extra- 
temporel. Un tel but ne peut être atteint que par ce qu'on pourrait nommer un oubli mythique $^{12}$ de la décision fondatrice dont l'ordre est né, l'oubli, par conséquent, de son historicité. Aussi, comme le souligne Eliade, le mythe consiste-t-il toujours en une révolte contre le temps historique ${ }^{13}$. Même si l'usage de la forme linguistique du futur antérieur semble transparent, il a le pouvoir de représenter l'histoire comme un processus consistant, prétendant connaître les secrets du passé, les dédales du présent, les incertitudes de l'avenir, ceci parce qu'il feint de connaître leur logique inhérente (je cite ici la définition donnée par Arendt de l'idéologie ${ }^{14}$ ). À son tour, la forme du futur antérieur voile le fait que la "logique inhérente » selon laquelle le développement historique de l'ordre politique est décrit est en fait installée par cet ordre lui-même. La démocratie dont la représentation est par principe toujours ouverte au débat ne peut éviter ceci non plus et se rapproche ainsi périlleusement de la «société sans histoire» qui est selon Lefort à la fois la société pré-moderne et la société totalitaire ${ }^{15}$. S'il y a une force impliquée dans la loi ${ }^{16}$, celle-ci réside avant tout dans la force du langage représentatif. Pour être bref, de même que selon l'adage bien connu, ce sont les vainqueurs qui écrivent les livres d'histoire, il est clair que ceux qui représentent la communauté déterminent ce qui compte comme histoire et, rétrospectivement, la légitiment. Aussi les livres d'histoire et les préambules des constitutions (sans parler des hymnes nationaux), peuvent-ils généralement être conçus comme des récits mythiques d'origine par excellence ${ }^{17}$.

Si nous voulons interrompre la logique mythique de l'ordre politique - et ceci est bien entendu l'enjeu ultime de l'analyse de Benjamin - nous devons donc avoir en vue cette dimension représentative. Aussi la critique de la violence faite par Benjamin est-elle, à travers cette analyse de la structure langagière du mythe, une motion de censure déposée contre la démocratie parlementaire en tant que système politique représentatif, précisément ${ }^{18}$. Selon Benjamin, le problème insurmontable de la démocratie représentative est que les mesures prises pour conserver l'ordre - celles de la police, des forces de l'ordre, des agences chargées de la lutte antiterroriste, etc. semblent être des exécutions neutres, tandis qu'elles témoignent toutes d'une violence latente. Alors que dans un ordre politique tyrannique et malveillant, on vit dans la pleine conscience de la présence latente de la violence dans toutes ses ordonnances, cette conscience risque de disparaître complètement dans une démocratie parlementaire. Dans nos démocraties, nous sommes, en d'autres termes, sur le point de perdre notre sensibilité vis-à-vis de la contingence, voire de l'arbitraire des mesures prises, et, par conséquent vis-à-vis de la responsabilité que cette contingence entraîne ${ }^{19}$. Malgré les avantages innombrables de la démocratie par rapport aux régimes tyranniques, la perte de cette sensibilité ne peut être conçue, selon Benjamin, que comme un signe sérieux de dégénérescence: "Schwindet das Bewußtsein [disparaisse la conscience] von der latenten Anwesenheit der Gewalt in einem Rechstinstitut, so verfällt es [périclite]» (Benjamin, 1977, p. 190. Je souligne).

\section{La rupture du cercle /ou l'interruption de la politique mythique}

Que faire, alors, pour contrecarrer cette tendance à la dégénérescence de nos démocraties contemporaines? Comment faire renaître notre sensibilité et reprendre notre responsabilité ? Selon Benjamin, il n'y a pas moyen de faire marche arrière, parce que cette perte de sensibilité tend par principe à être totale : le raisonnement mythique menant 
à l'oubli mythique est nécessairement auto-immunisant, selon un mot de Derrida ${ }^{20}$. Comme je l'ai indiqué, la structure même de l'ordre est telle qu'une vraie interrogation sur ses fondements est impossible. Certes, dans une démocratie, les lois établies et même les actes des politiciens sont continuellement soumis au débat. Une constitution démocratique prévoit même officiellement la possibilité de sa propre modification. Une telle procédure de révision ne concerne toutefois que la correction des imperfections ou la modification des règles individuelles. Une fois instauré, l'ordre politique en tant que tel ne peut plus être officiellement remis en question.

19 Selon Benjamin, ceci se démontre de façon exemplaire dans le cas du droit de grève. Dans la plupart des démocraties actuelles, le droit de grève s'accompagne d'un encadrement réglementaire ou législatif et est généralement perçu comme une caractéristique fondamentale de la démocratie. Cependant, ce droit de grève ne concerne, selon lui, que ce qu'il nomme à la suite de Georges Sorel la grève générale politique. N'ayant pour but que la modification de certaines conditions de travail, cette forme de grève ne remet pas en question l'ordre politique en tant que tel, mais mène au contraire à son renforcement. Cette forme de grève se distingue de ce qu'il nomme la grève générale prolétarienne ${ }^{21}$. Dans ce dernier cas, les grévistes ne se contentent pas d'une modification des détails, mais invoquent leur droit de grève en aspirant à la chute de l'État. Ils n'ont donc pas pour but de parvenir à telle ou telle fin déterminée, à telle ou telle situation idéologique, mais veulent seulement se défaire de l'ordre donné pour ouvrir de nouvelles perspectives (Benjamin, 1977, p. 193-194). C'est pourquoi Benjamin qualifie également cette grève de "révolutionnaire » ou "anarchiste». Alors que la grève politique renforce l'ordre existant plutôt qu'elle ne le menace, la grève révolutionnaire, elle, entraîne son ébranlement. Au moment où une grève politique risque de se transformer en grève révolutionnaire, comme ce fut le cas par exemple en Grèce en décembre 2008, les détenteurs du pouvoir se hâtent de procéder à des mesures exceptionnelles pour conserver l'ordre tel qu'il est établi - et donc leur pouvoir - en déclarant « illégitime » le droit de grève normalement légitime et instauré précisément pour des raisons démocratiques ${ }^{22}$.

20 Ce que démontre l'exemple du droit de grève, c'est que même si un ordre politique, contient, comme nos démocraties actuelles, la possibilité de sa propre contestation et, par conséquent, de la remise en question de son idée et de sa forme, il est prêt à violer ce principe pour assurer son pouvoir. C'est pourquoi Benjamin ne voit qu'une seule solution pour nous réveiller de notre oubli mythique et nous faire retrouver notre sensibilité à la faillibilité des représentations politiques de nos communautés : ouvrir une nouvelle ère historique où nous nous déferions complètement de l'organisation des communautés sous forme d'États de droit. Cette nouvelle ère historique doit se fonder

«auf die Durchbrechung [rupture] dieses Umlaufs im Banne der mythischen Rechtsformen, auf der Entsetzung [destitution] des Rechts samt den Gewalten, auf die es angewiesen is wie sie auf jenes, zuletzt also der Staatsgewalt » (Benjamin, 1977, p. 202. Je souligne).

21 Cette Durchbrechung, cette rupture - et ceci est sans doute l'élément à la fois le plus difficile et le plus fascinant du «diagnostic» de Benjamin - doit se réaliser par une manifestation de ce que Benjamin nomme la «violence divine». Mais qu'est-ce que cette mystérieuse violence qualifiée de divine ou encore de pure ou d'anarchiste? Elle n'est pas, comme par exemple la grève politique, une forme de contestation dirigée contre quelque 
chose de spécifique. Il s'agit plutôt d'une contestation pour rien qui résiste pour cette raison à une réappropriation dans le circuit instrumental des moyens et des fins.

Même si la description benjaminienne de l'interruption de la politique mythique est souvent perçue comme un plaidoyer pour un anarchisme radical, voire sanglant et destructeur, le cœur de cette interruption anarchiste réside à mon avis non pas dans sa portée destructrice, mais dans le fait qu'elle s'abstient de toute décision, ou plus précisément, de toute décision politique. Toute décision politique, suggère Benjamin, est la détermination des rapports moraux aboutissant à la détermination des frontières, à trancher - entre ce qui est légitime et ce qui ne l'est pas, entre ce qui fait partie de l'ordre et ce qui n'en fait pas partie, etc. ${ }^{23} \mathrm{Ne}$ pas décider de façon politique, pour Benjamin, ne revient pas à s'abstenir de décider tout court, mais à s'abstenir de faire des décisions qui se légitiment par la référence à un destin historique. Car c'est seulement par référence à un destin historique que « die Grenzsetzung [devient] das Urphänomen rechtsetzender Gewalt » (Benjamin, 1977, p. 198). C'est à dire que les actes qui fondent ou conservent le droit déterminent nécessairement des frontières en vue d'un destin et entrent par conséquent dans une logique s'appuyant sur des notions comme la responsabilité, le sacrifice, la dette et la punition.

Mais comment s'abstenir de décider de facon politique? La réponse aussi simple qu'énigmatique de Benjamin est donc: Vernichtung [l'anéantissement ${ }^{24}$. Il est important de noter qu'il ne s'agit pas nécessairement, à mon avis, d'un anéantissement dans le sens de la destruction de l'ordre donné, c'est-à-dire de l'infrastructure, des organes vitaux ou des vies humaines, comme celle que visent par exemple les terroristes. Il s'agit bien en revanche d'une Vernichtung : un réduire à rien [nichts], un rendre nihil, une annulation de la configuration donnée. Non pas une reconfiguration du donné, mais l'ouverture d'un ailleurs. Comme Benjamin l'a élaboré dans "Der destruktieve Charakter", un tel anéantissement ne se produit pas tellement "par amour des décombres", mais "par amour pour le chemin qui se fraie un passage à travers eux ", autrement dit, pour les nouvelles perspectives qui pourraient s'ouvrir (Benjamin, 1977). Il s'agit donc de la création d'une forme d'épochè où le « cercle magique des formes mythiques du droit » est momentanément rompu, où la couronne construite au nom du destin est momentanément levée et où on peut apercevoir l'au-delà de l'ordre donné. ${ }^{25} \mathrm{La}$ perspective ouverte n'est donc pas celle d'un autre ordre politique, mais de l'autre de la politique, d'un "ordre supérieur ", divin, où les décisions ne sont pas alourdies par la couronne du destin, mais témoignent d'une perméabilité et mobilité permanentes.

\section{Un mot contre le droit / ou l'impunité du mensonge}

Il importe de noter qu'une telle interruption n'est pas quelque chose qu'on peut calculer ou prévoir. Aussi, le terme divin indique-t-il qu'elle n'est pas contrôlable, qu'elle ne fait pas partie d'une logique instrumentale, mais qu'elle frappe sans avoir averti (Benjamin, 1977, p. 199). C'est-à-dire aussi qu'elle n'est pas entre nos mains : ça arrive. En outre, l'assertion selon laquelle cette " nouvelle ère historique » va arriver ferait immédiatement retomber la tentative d'interrogation en une logique de la violence mythique. Néanmoins, il y a, selon Benjamin, des indices qui témoignent de la possibilité d'une telle interruption. La nouvelle ère semble donc toujours être déjà potentiellement présente et capable de commencer à chaque instant ${ }^{26}$. Ceci est aussi suggéré par Benjamin quand il dit, au conditionnel, que «wenn die Herschaft des Mythos hie und da im Gegenwärtigen schon 
gebrochen ist, so liegt jenes Neue nicht in so onvorstelbarer Fernflucht, da $\beta$ ein Wort gegen das Recht sich von selbst erledigte » (Benjamin, 1977, p. 202). ${ }^{27}$

La formulation exacte importe ici. Ce qui fut traduit en français comme l'objection éventuelle contre le droit qui pourrait interrompre le règne du mythe est, selon Benjamin, ein Wort gegen das Recht, un mot donc, un mot qui peut se tourner contre le droit. Le détail de cette formulation semble anecdotique, mais ce détail dévoile toute sa portée si l'on s'attache à l'idée du langage qu'il implique. En effet, comme nous l'avons déjà brièvement évoqué, selon Benjamin la dégénérescence particulière de nos démocraties contemporaines va de pair avec une certaine dégénérescence de notre usage du langage. Comme il le suggère, avant que nos démocraties commencent à dégénérer, la rationalité instrumentale n'avait pas encore pénétré tous les domaines de la vie et il y avait encore « eine in dem Grade gewaltlose Sphäre menschlicher Übereinkunft [die] der Gewalt volständig unzugänglich ist : die eigenliche Sphäre der 'Verständigung' [l'entente], die Sprache [langage] » (Benjamin, 1977, p. 192). Le domaine du langage, autrement dit, n'était pas encore entièrement politisé et faisait place à une sphère neutre d'entente, de communication. Il s'agit ici d'une sphère de communication qui n'est pas régie par des catégories juridico-politiques comme la légitimité et l'illégitimité, mais qui se caractérise par le dialogue, ce dialogue qui est, selon Benjamin la « Technik ziviler Übereinkunft » (Benjamin, 1977, p. 191-192). Parce qu'il ne s'agit pas d'une technique juridico-politique, la liquidation des conflits éventuels se passe dans cette sphère, à strictement parler sans violence. L'important est que, dans ce domaine de l'entente, il ne s'agit pas seulement de s'entendre sans violence, mais il est même question d'une "prinzipielle Ausschaltung [ exclusion] der Gewalt »(Benjamin, 1977, p. 192. Je souligne).

Le fait qu'elle exclut la violence signifie que cette technique d'entente n'aspire pas à fonder ou à conserver du droit. Dans la sphère de la communication, on ne fait pas appel au droit, mais à la sympathie, à l'amour ou à la confiance (Benjamin, 1977, p. 191). Autrement dit, dans une sphère de communication véritable, on a recours à ces moyens parce qu'aucune des parties n'a le pouvoir de forcer une décision. Il s'agit donc ici, comme le remarque justement Antonia Birnbaum, d'une sphère hétérogène où le langage est moins un «moyen de communiquer un contenu ou de vérifier le vrai et le faux d'un référent objectivé » qu'« une médiation située par-delà cette différence » (Birnbaum, 2008, p. 66 et 74). Qu'on ne prenne pas le pouvoir de forcer une décision veut dire qu'on ne revendique aucune validité. Si la parole n'aspire pas à fonder ou conserver du droit, "so verzichtet sie damit selbst auf jede Geltung " : elle renonce d'elle-même à toute validité28. Il en découle - et ceci est à mon avis un élément extrêmement important mais trop peu remarqué de l'analyse de Benjamin - qu'une prise de parole est «non violente » si elle renonce d'elle-même à toute validité.

La preuve la plus claire du fait que nos démocraties contemporaines sont "dégénérées » et que cette dégénérescence va de pair avec une dégénérescence de l'usage de notre langage est, selon Benjamin, précisément le changement de notre attitude envers le mensonge. Le fait qu'à l'origine, aucune législation n'ait puni le mensonge, constitue une preuve pour Benjamin qu'il existait effectivement un domaine du langage non violent à l'abri du droit. À l'époque, les conflits éventuels causés par le (soupçon de) mensonge étaient aux risques et périls du peuple, en vertu du principe que «le droit civil a été écrit pour des vigilants [jus civile vigilantibus scriptum est]» (Benjamin, 1977, p. 192). La parole mensongère n'était donc pas conçue comme une attaque contre l'ordre du droit, mais seulement comme un conflit d'intérêts devant se régler par l'entente mutuelle. 
L'impunité du mensonge, dans les ordres politiques antiques, est donc pour Benjamin le signe de leur vitalité et de leur confiance en eux-mêmes comme en la vigilance du peuple.

$\mathrm{Au}$ cours des temps, nos démocraties parlementaires ont néanmoins commencé à inclure le mensonge, et par extension la tromperie et la mystification, dans l'usage politique du langage et dans la sphère d'exercice du droit. Avec la conséquence inévitable de les punir. Ou comme le dit Benjamin :

« Erst spät und in einem eigentümlichen Verfallsprozeß [un processus caractéristique de décadence] ist die Rechtsgewalt dennoch in sie [le domaine propre à l'entente, celui du langage, AvR] eingedrungen, indem sie den Betrug [la tromperie] unter Strafe stellte. »(Benjamin, 1977, p. 192) ${ }^{29}$

En quoi cette intrusion de l'État dans la sphère de l'entente non violente est-elle un signe de décadence ou de dégénérescence ? L'introduction du droit dans tous les domaines de la vie n'est-elle pas le signe d'une progression juridique ou morale? Ne témoigne-t-elle pas du fait que l'État se sent responsable de ses citoyens et les préserve de subir des dommages? Selon Benjamin, juger que le pouvoir de déterminer si telle ou telle parole relève de la tromperie doit être placé entre les mains de l'État et non relever d'une réflexion autonome de l'individu mène inévitablement à la perte de sensibilité vis-à-vis de la présence latente de violence dans l'institution juridique. Si l'institution s'en prend à la tromperie, ce n'est pas pour des motifs moraux, mais par crainte de l'affaiblissement de l'ordre politique que cette tromperie pourrait entraîner (Benjamin, 1977, p. 192). Les deux affaires néerlandaises mentionnées dans l'introduction attestent éloquemment de cette crainte.

Le paradoxe est que si l'expansion de la sphère d'influence du droit dans le domaine du langage a pour but son renforcement, elle entraîne néanmoins le risque de son affaiblissement. On pourrait même aller plus loin et avancer qu'elle entraîne non seulement le risque de l'affaiblissement de nos démocraties, mais, à long terme, celui de leur auto-déconstruction. Cette expansion de la sphère d'influence de l'État témoigne pourtant, selon Benjamin, d'une forte «Mißtrauen [méfiance] in sich selbst » (Benjamin, 1977, p. 192). En effet, l'État considère précisément comme illégitimes les pratiques de débat qui ne se prévalent pas d'une justification décisive de leur légitimité. Le souci de Benjamin est que nos démocraties risquent de diminuer la vitalité de la société, c'est-àdire sa capacité à se développer et à se modifier, à remettre en question ses idées fondatrices. Un ordre vital est, selon lui, un ordre qui ne craint pas de faire place aux pratiques qui se soustraient à la portée du droit, même si celles-ci peuvent éventuellement évoquer une violence dirigée contre l'État.

31 À mon avis, Benjamin a ainsi préparé la thèse défendue par Derrida et plus tard par Roberto Esposito et par Jean-Luc Nancy : à savoir que le risque le plus grand pour l'ordre politique est celui de son auto-immunisation ${ }^{30}$, c'est-à-dire le risque qu'en essayant de se protéger excessivement contre des attaques néfastes, l'ordre ne se détourne des éléments vitaux pour sa survie - survie qui pourrait-on dire est néanmoins celle d'un mort-vivant. La source de la vitalité d'un ordre semble pourtant être le "vide » dans lequel non seulement s'est érigé l'ordre donné, mais auquel renvoient également ceux qui l'interrogent. Ce vide a pour conséquence que la validité de l'ordre établi n'est pas à imposer, mais qu'elle est impérative comme «Richtschnur [fil conducteur] » de l'ordre politique, dont on doit constamment assumer la responsabilité, quitte à ne pas tenir compte de cet ordre si c'est nécessaire (Benjamin, 1977, p. 200-201) ${ }^{31}$. 


\section{Un potentiel subversif / ou parler sans pouvoir}

\section{piste de réflexion immédiatement refermée par lui et largement négligée par les} nombreux commentateurs de son texte. L'importance de cette piste réside dans le fait qu'elle indique une forme d'interruption du cercle mythique qui ne vient pas d'un au-delà de l'ordre de droit, ni ne l'exige, mais est originairement acceptée et préservée dans cet ordre même, une forme de communication non politisée dans l'ordre politique. Benjamin lui-même, me semble-t-il, referme cette possibilité par son manque de confiance en les démocraties contemporaines ou, pour être plus précis, par son manque de confiance en la confiance qu'elles ont en elles-mêmes. Selon Benjamin, la politique mythique est le résultat d'un processus de dégénérescence caractéristique de nos États contemporains. La démocratie parlementaire diffère selon lui des ordres politiques antérieurs, parce que sa structure représentative rend possible l'oubli mythique total, c'est-à-dire la perte de toute conscience de la violence de la décision originaire, perte de conscience entretenue par la conservation de notre ordre. Ce risque révèle, selon Benjamin, qu'il y a « etwas Morsches [quelque chose de corrompu] » (Benjamin, 1977, p. 188) dans nos ordres de droit non pas dans leur disposition extérieure, mais dans leur cœur même. Répétons-le : quelle que soit la bonne volonté du gouvernement ou du peuple, toute véritable mise en question de l'idée et de la forme de l'ordre politique est pour cette raison rendue impossible selon Benjamin.

Par conséquent, la seule manière de susciter une véritable interrogation est pour lui de se défaire complètement de cette structure politique de l'ordre de droit. Pour pouvoir réellement assumer la tâche de nous constituer nous-mêmes comme communauté et d'en assumer la pleine responsabilité, il nous faut, d'après lui, une autre forme de politique. Cette autre forme de politique ne consisterait pas en un enchevêtrement des moyens et des fins, mais devrait être une " politique des moyens purs », une politique où les moyens n'ont pas de but hors d'eux-mêmes (Benjamin, 1977, p. 193) ${ }^{32}$. Dans ce cas, il ne s'agirait même pas d'un ordre, mais, comme le dit Birnbaum, d'un « désordre qui ne tolère plus aucune hiérarchie en son sein » (Birnbaum, 2008, p. 73), et où l'on vit à chaque moment dans le présent. Au lieu de la temporalité mythique du futur antérieur pour ainsi dire «flottante ", ce « désordre » ne connaîtrait pas de destin couronné, ni d'apparence de continuité, ni même de progression quelconque, mais consisterait pour ainsi dire en une suite éternelle d'instants présents ${ }^{33}$. Car, comme le suggère Benjamin, c'est seulement ainsi qu'on peut assumer la responsabilité d'un ordre politique post-fondationaliste : une responsabilité consistant à accepter qu'à chaque moment quelque chose de radicalement nouveau puisse arriver.

Bien que cette façon de penser un être-ensemble indépendant des structures étatiques et nationales soit intéressante, on peut se demander si une politique du présent est à strictement parler à éviter. Ne devrait-on pas soutenir que l'indécidabilité du futur est en même temps ce qui contraint au politique? Comme le suggère Benjamin, la dimension mythique fait partie de la structure du politique et c'est pour cette raison qu'il met son espoir dans un ordre supérieur non-politique, dans une autre ère historique. Comme nous l'avons vu, son recours à l'idée d'un ordre supérieur résulte, entre autre, de son manque de confiance en la vitalité des démocraties contemporaines. J'aimerais néanmoins étudier la possibilité d'un ordre politique, c'est-à-dire mythique, vital, ne serait ce que parce que 
le cercle mythique me semble, pour le moment, inévitable. Rappelons-nous donc le diagnostic que fait Benjamin quant à la vitalité d'un ordre politique. Ce que l'analyse de Benjamin nous apprend est que l'absence de vitalité d'un ordre politique se laisse mesurer par son manque de confiance en soi, et ce manque de confiance se traduit par une immunisation croissante de la logique mythique, qui, à son tour, se laisse mesurer par une pénétration de la logique politique dans un nombre croissant de domaines de la vie. Inversement, la vitalité d'un ordre de droit est reconnaissable au fait qu'il fait place aux domaines qui se trouvent hors de son influence.

Mais une politique mythique vitale est-elle possible ? Pour commencer, il faut se rappeler qu'à l'état de germe, cette vitalité est déjà présente dans la structure mythique ellemême. Comme je l'ai indiqué, la structure du futur antérieur non seulement déclare que les choses auront été ainsi, mais révèle en même temps qu'aucune certitude ne garantit cette déclaration. Parce que le cercle mythique consiste en une fiction fondatrice, son interruption éventuelle n'est pas une interruption qui a lieu pour ainsi dire après-coup, mais une interruption qui a toujours déjà lieu dans cette construction paradoxale mythique elle-même. La possibilité de son interruption est donc impliquée dès la constitution de la politique mythique, ou plutôt par sa constitution. Ceci n'empêche pourtant pas que la logique mythique tende, volens nolens, vers ce que nous avons nommé l'oubli mythique, comme nous en prévient Benjamin. Il importe donc de maintenir d'une manière ou d'une autre la conscience de et la sensibilité pour la dimension mythique de la politique. Mais si la logique mythique est nécessairement immunisante, comment alors, de l'intérieur de l'ordre politique, maintenir cette conscience ? La valeur de l'analyse de Benjamin - et ici nous retournons à la piste ouverte par l'excursus sur l'impunité du mensonge - est non seulement qu'elle met au jour la parenté de nos ordres et des sociétés mythiques, mais aussi qu'elle soulève un coin du voile qui recouvre le fonctionnement langagier sous-jacent à cette parenté.

Ce qui importe ici est l'observation faite par Benjamin que les expressions qui s'abstenaient d'une décision politique pouvaient originairement compter sur une place hors de la sphère d'influence de l'État. Inversement, ce sont les expressions qui revendiquent la validité qui sont conçues comme violentes et éventuellement comme menaçantes, parce que ce sont ces expressions qui décident des rapports moraux valides au sein de l'ordre politique. Bien que l'annexion de nouveaux domaines sociaux par la politique mythique soit en effet un signe de son immunisation, cela n'ôte rien à l'observation que c'est dans l'abstention à la prétention de validité que se trouve un potentiel non-politique possible. Il s'agit donc de découvrir où se trouve actuellement ce potentiel qui appartenait autrefois au domaine de l'entente mutuelle. Si l'on prend au sérieux le diagnostic pessimiste de Benjamin vis-à-vis de nos États, il faut pour remettre en question l'idée et la forme de l'ordre donné sans se défaire entièrement de l'État, recourir aux expressions qui ne laissent aucun doute quant à leur abstention de toute décision politique, c'est-à-dire quant au fait qu'elles ne se réfèrent à aucune forme de légitimité et sont faites pour ainsi dire ex nihilo et pour rien. Ce dernier aspect est à mon avis crucial. Car les actes des grévistes prolétariens, pour répéter cet exemple-clé de Benjamin, ne sont pas bâillonnés parce qu'ils interrogent l'ordre donné, mais parce que leur interrogation est considérée comme menacante. C'est pour cela que ces actes sont immédiatement réabsorbés dans la logique mythique. Autrement dit, ce n'est pas l'interrogation, mais la menace qui semble être inacceptable pour un ordre politique. Bien évidemment, c'est l'interrogation des fondements de l'ordre qui est menaçante, mais 
l'une n'est néanmoins pas entièrement réductible à l'autre. Aussi est-ce sans doute surtout l'estimation du caractère menaçant et non pas le genre de l'interrogation luimême qui a changé au cours du temps.

Aujourd'hui, le potentiel subversif ne réside peut-être plus tellement dans l'action directe, dans la proclamation d'un «autre » monde " plus vrai », mais dans l'abstention de proclamation et de programmatique des aspirations révolutionnaires. On pourrait conclure que ce potentiel réside surtout dans une forme de communication nonlangagière, dans l'interaction des corps muets ${ }^{34}$. Mais comme l'excursus sur l'impunité de la tromperie mensongère le laisse voir, on peut également chercher cette subversion dans une forme de parole muette, dans une parole qui préfère se taire quant à sa validité, et qui est peut-être surtout la parole trompeuse et mensongère de l'art et de la littérature.

À l'opposé de la parole politique du détenteur du pouvoir et de la parole contre-politique des grévistes et des activistes, la parole de l'artiste ne veut pas revendiquer la validité et renonce même explicitement à le faire. Comme nous venons de le constater avec Benjamin, une telle revendication de validité est peut-être le moteur qui met en mouvement la logique mythique selon laquelle se structure un ordre politique. Si une interruption du cercle mythique de nos ordres politiques est possible, elle réside donc dans une tentative de faire place, dans nos ordres politiques, à un genre d'énonciations «trompeuses », «mensongères » ou "littéraires », qui, en cette qualité, mettent en question l'ordre donné sans en revendiquer un autre. Peut-être est-ce seulement ainsi que la mise en intrigue politique se laisse dénouer.

\section{BIBLIOGRAPHIE}

Abensour Miguel, De la compacité. Architecture et régimes totalitaires, Paris, Sens \& Tonka, 1997

Anderson Benedict, Imagined Communities. Reflections on the Origin and Spread of Nationalism, London/New York, Verso, 2006 (1983)

Arendt Hannah, The Origins of Totalitarianism, San Diego, Harcourt Brace Jovanovich, 1973 (1951)

Aristote, La poétique, trad. Roselyne Dupont-Roc \& Jean Lallot, Paris, Seuil, 1980

Benjamin Walter, « Der destruktive Charakter », Gesammelte Schriften, IV.1, Francfort, Suhrkamp, p. 396-398, 1977 (1931)

Benjamin Walter, « Schicksal und Charakter », Gesammelte Schriften, II.I, Francfort, Suhrkamp, p. 171-179, 1977 (1919)

Benjamin Walter, « Zur Kritik der Gewalt », Gesammelte Schriften, II.1, Francfort, Suhrkamp, p. 179-203, 1977 (1921)

Birnbaum Antonia, Bonheur Justice. Walter Benjamin, Paris, Payot, 2008

Butler Judith, « Critique, Coercion, and Sacred Life in Benjamin's 'Critique of Violence' », in Hent de Vried (éd.), Political Theologies. Public Religions in a Post-Secular World, New York, Fordham University Press, p. 202-219, 2006 
Le comité invisible, L'Insurrection qui vient, Paris, La Fabrique, 2007

Derrida Jacques, Psyché. Inventions de l'autre, Paris, Galilée, 1987

Derrida Jacques, Force de loi. Le « fondement mystique de l'autorité », Paris, Galilée, 2005 (1994)

Eliade Mircea, Le mythe de l'éternel retour. Archétypes et répétition, Paris, Gallimard, 1989

(1969)

Fenves Peter, Arresting Language. From Leibniz to Benjamin, Stanford, Stanford University Press, 2001

Girardet Raoul, Mythes et mythologies politiques, Paris, Seuil, 1986

Lefort Claude, «La question de la démocratie » in Jean-Luc Nancy \& Philippe Lacoue-Labarthe (éds.), Le retrait du politique, Paris, Galilée, 1983, p. 71-88

Marchart Oliver, Post-Foundational Political Thought. Political Difference in Nancy, Lefort, Badiou and Laclau, Edinburgh, Edinburgh University Press, 2007

Menninghaus Winfried, « Walter Benjamin's Theory of Myth » in Gary Smith (éd.), On Walter Benjamin. Critical Essays and Recollections, Massachusetts, MIT Press, 1988, p. 292-325

Nancy Jean-Luc, «L'impossible acte constituant », Le Monde, 29 juillet 2005

«Préambule », Traité établissant une constitution pour l'Europe, Rome, 29 octobre 2004

Proust Françoise, L'Histoire à contretemps. Le temps historique chez Walter Benjamin, Paris, Cerf, 1994

Ricoeur Paul, Temps et récit I. L'intrigue et le récit historique, Paris, Seuil, 1983

Sorel Georges, Réflexions sur la violence, Paris, Rivière, 1919

Rooden Aukje van, « Interrupting Mythological Politics? On the Possibility of a Literary Intervention », Theory \& Event, $12: 2,2009$

Wilde Marc de, Verwantschap in extremen. Politieke theologie bij Walter Benjamin en Carl Schmitt, Amsterdam, Amsterdam University Press, 2008

\section{NOTES}

1. La question du mensonge de Hirsi Ali se complique encore quand on considère que si selon le droit néerlandais cette dernière a bien menti sur son nom de famille, selon le droit somalien en revanche, il n'est pas interdit de porter le nom de son grand-père au lieu de celui de son père, comme l'a fait Hirsi Ali lors de son arrivée.

2. En 2004, Ayaan Hirsi Ali réalisait avec le cinéaste Theo van Gogh un court-métrage militant sur la pénibilité de la condition féminine dans le monde musulman. Van Gogh fut assassiné par un extrémiste musulman. L'assasin planta un couteau dans la poitrine de Van Gogh en y épinglant une lettre adressée à Ayaan Hirsi, et la désignant comme la future victime du poignard vengeur.

3. Une autre affaire plus médiatique dans ce cadre est "l'affaire Lewinsky " aux États-Unis, menant à la procédure d'impeachment pour parjure du président américain Bill clinton.

4. D'abord publié dans Archiv für Sozialwissenschaft und Sozialpolitik, 1921, et repris dans Walter Benjamin, Gesammelte Schriften II.1. Traduit en français sous le titre «Pour une critique de la violence » par M. de Gandillac dans Walter Benjamin, Mythe et violence, 1971, repris dans L'Homme, le langage et la culture, 1974, puis sous le titre «Critique de la violence » dans Walter Benjamin, 
CEuvres I. Je me réfère à l'édition Gesammelte Schriften. Je me réfère toujours aux versions originales des textes cités, que je compare si nécessaire avec la traduction française.

5. Le caractère déséquilibré de ma lecture réside en partie dans le fait que je ne prendrai pas ou seulement très brièvement en considération certains passages importants de ce texte. Voir pour une lecture plus élaborée (Van Rooden, 2009)

6. Cf. (Benjamin, 1977, p. 181 et 202) où Benjamin parle respectivement d'un «Zirkel » et d'un «Umlauf ». Dans son analyse du texte de Benjamin, Derrida suggère que Benjamin lui-même ne s'est pas explicitement rendu compte de cet entrelacement circulaire de la violence fondatrice et de la violence conservatrice : «[A] $\mathrm{u}$-delà du propos explicite de Benjamin, je proposerai l'interprétation suivant laquelle la violence même de la fondation ou de la position du droit ( Rechtsetzende Gewalt) doit envelopper la violence de la conservation du droit (Rechtserhaltende Gewalt) et ne peut pas rompre avec elle. Il appartient à la structure de la violence fondatrice qu'elle appelle la répétition de soi et fonde ce qui doit être conservé, conservable » (Derrida, 2005, p. 93). Bien que je ne pense pas que cette interprétation mène au-delà du propos de Benjamin, Derrida a à mon avis raison de souligner qu'il s'agit ici d'un entrelacement structurel inévitable et non d'un entrelacement résultant d'un certain déclin historique comme le suggère Benjamin.

7. Pour une analyse du rôle central de la notion de destin dans «Zur Kritik der Gewalt », voir aussi (Birnbaum, 2008)

8. Cf. (Aristote, 1980, chapitre 7). La traduction par «mise en intrigue » est de Paul Ricoeur (Ricoeur, 1983). Benjamin lie également la notion de destin à la tragédie, dans son « Begriff des Schicksals im Schicksaldrama », Ursprung des deutschen Trauerspiels. Voir aussi le chapitre "La chronique des temps présents » dans (Proust, 1994).

9. De façon moins explicite, l'appel au progrès en vue d'une certaine fin déterminée est également mis en œuvre dans le préambule du traité établissant une constitution pour l'Europe : "Convaincus que l'Europe, désormais réunie au terme d'expériences douloureuses, entend avancer sur la voie de la civilisation, du progrès et de la prospérité [...] 》 ('Préambule', 2004).

10. Dans mon interprétation, je vais plus loin que Judith Butler, qui décrit l'acte constituant comme " the claim that 'This will be law' or, more emphatically, 'This is now the law' (Butler, 2006, p. 202). Dans la terminologie de Butler, je dirais donc: "This will always have been the law ».

11. La double signification du futur antérieur est entre autres indiquée par Jacques Derrida (Derrida, 1987, p. 190).

12. Le terme d'« oubli mythique » est de Marc de Wilde (De Wilde, 2008, p. 125sq). Cf. aussi (Derrida, 2005, p. 113), qui parle d'une «dénégation amnésique». Benjamin parle d'un «schwinden [disparaître] des Bewußtseins » (Benjamin, 1977, p. 190).

13. Cf. (Eliade, 1989), notamment le chapitre « La 'terreur de l'histoire' ».

14. "The ideology treats the course of events as though it followed the same 'law' as the logical exposition of its 'idea'. Ideologies pretend to know the mysteries of the whole historical process, the secrets of the past, the intricacies of the present, the uncertainties of the future-because of the logic inherent in their respective ideas. [It is] the 'idea' by which the movement of history is explained as one consistent process. » (Arendt, 1973, p. 469)

15. Lefort emprunte cette notion de "société sans histoire" à Hegel (Lefort, 1983, p. 80). Selon Lefort, un système totalitaire est une telle société non-historique. Selon lui, un système totalitaire est analogue à une société primitive dans le sens où les deux opèrent une dénégation du rôle du pouvoir en vue de préserver leurs communautés. Comme le souligne Lefort, un système totalitaire non seulement ignore sa condition historique, mais la réprime activement en créant une société où tout est déterminé une bonne fois pour toutes et où sont abolies les circonstances dynamiques dans lesquelles l'action politique prend place. Sur la non-historicité, voir aussi (Girardet, 1986, p. 101): « [L]e troisième palier de la construction mythique : celui de la non-histoire. »; et 129: « Le monde de l'Age d'or est celui des horloges arrêtées. » La non- 
historicité de la société mythique peut également être exprimée en terme cyclique de l'éternel retour, comme le fait Eliade. Cf. (Eliade, 1989).

16. Comme le suggère Derrida avec le titre de son livre sur le fondement "mystique» de l'autorité, Force de loi. Comme le mot Gewalt en allemand, le mot force indique qu'il ne s'agit pas ou pas seulement d'un pouvoir autorisé, officiel, mais toujours aussi d'une forme de pression.

17. C'est pourquoi on peut dire à juste titre que les livres d'histoires sont comme des romans nationaux, comme le propose (Anderson, 2006).

18. Cf. aussi (Derrida, 2005, p. 69).

19. Ainsi est-il largement douteux que la démocratie occidentale contemporaine puisse sans accroc être exportée et imposée, par exemple dans les pays du Moyen-Orient.

20. Cf. par exemple (Derrida, 1993) et (Derrida, 1996).

21. La distinction entre la grève politique et la grève prolétarienne se lit dans le septième alinéa de son texte, mais il reprend les termes de Sorel seulement dans le treizième alinéa. Cf. (Sorel, 1919).

22. Plus précisément, les détenteurs du pouvoir le déclarent illégitime en disant que le droit de grève «n'a pas été entendu 'ainsi' » (Benjamin, 1977, p. 184). Une fois de plus nous voyons que le pouvoir réside dans le pouvoir de représenter, in casu l'« ainsi ».

23. Cf. aussi (Proust, 1994, p. 136) : «L'essence de l'État, et par suite, du politique, n'est pas la loi (le droit) ni la volonté (la subjectivité législatrice), elle est la décision. [...] La décision comme capacité de trancher est l'essence du politique ». Elle se fonde sur (Benjamin, 1977, p. 198) : «In seinem Bereich [des Staatsrechtes, AvR] ist die Grenzsetzung [...] das Urphänomen rechtsetzender Gewalt überhaupt ».

24. Lisons le passage-clé de «Zur Kritik der Gewalt »: «Ist die mythische Gewalt rechtsetzend, so die göttliche rechtsvernichtend, setze jene Grenzen, so vernicthtet diese grenzenlos, ist die mythische verschuldend und sühnend zugleich, so die göttliche entsühnend» (Benjamin, 1977, p. 199). Parce que le caractère violent d'un acte réside dans la détermination des rapports moraux, comme le dit Benjamin dans les premières phrases de son texte, on peut même dire que les actes de violence divine ne sont pas violents.

25. Cf. aussi (Fenves, 2001), notamment " The Paradisal Epoche. On Benjamin's First Philosophy ». 26. Cf. (Menninghaus, 1988).

27. La traduction française : "Si déjà le règne du mythe est présentement, ici et là, battu en brèche, ce nouveau ne se situe pas dans un horizon lointain si difficile à concevoir qu'une objection contre le droit se règlerait d'elle-même » (Benjamin, 2000, p. 242).

28. «Alle Gewalt ist als Mittel entweder rechtsetzend oder rechtserhaltend. Wenn sie auf keines dieser beiden Prädikate Anspruch erhebt, so verzichtet sie damit selbst auf jede Geltung " (Benjamin, 1977, p. 192. Je souligne).

29. Miguel Abensour a nommé cette pénétration dans tous les domaines de la vie la «compacité» de l'architecture mythique moderne qui produit un espace sans écart (Abensour, 1997). Cf. aussi (Birnbaum, 2008, p. 81). Et en effet, nous pouvons apercevoir, dans nos démocraties contemporaines, une tendance à intervenir contre un nombre croissant de formes de parole mensongère ou trompeuse. Pensons par exemple aux interdictions et mesures contre la publicité mensongère, contre les photos manipulées, contre des caricatures provocatrices.

30. Cf. (Esposito, 2000) et (Esposito, 2005); (Nancy, 1986) et (Nancy, 2000).

31. Selon Benjamin, une telle responsabilité est exigée par le commandement biblique «Tu ne tueras pas ». Pour une analyse concise du rôle de ce commandement dans le texte de Benjamin, voir (Butler, 2006, p. 205).

32. Il faut bien noter que ces "moyens purs" ne doivent pas être compris de façon aristotélicienne, comme le remarque aussi Peter Fenves : « The idea of a pure means [...] cannot issue into that of a 'means in itself', for something can be declared to be 'in itself' only on the condition that it is, in the Aristotelian sense 'perfect,' which is to say, at its end. One can speak of 
a pure means if the end in view of which means are defined as means is not so much nullified or emptied of content as indefinitely delayed, extended, distended, or enfolded on - rather that 'in' - itself » (Fenves, 2001, p. 253).

33. Sur l'expérience du temps chez Benjamin cf. "Arrêter le jour» dans (De Wilde, 2008, p. 125-179).

34. Comme le suggère Benjamin dans Ursprung des deutschen Trauerspiels, en se référant au héros tragique aux prises avec le destin, ainsi qu'en un sens Antonia Birnbaum dans (Birnbaum, 2008).

\section{RÉSUMÉS}

Cette contribution part de l'hypothèse que ce ne sont pas tant les ordres politiques pre-modernes que nos ordres politiques contemporains qui font appel à une logique mythique. N'ayant pas d'autorité externe, nos ordres politiques s'érigent nécessairement à partir d'un mouvement d'auto-constitution. En référence à la «Critique de la violence» de Walter Benjamin, une telle constitution peut être conçue comme un enchevêtrement mythique de fondation et de conservation. Ce cercle mythique referme la possibilité d'une mise en question fondamentale de l'ordre existant, en dépit de la volonté caractéristique des régimes démocratiques de s'exposer à un débat sans fin. C'est pourquoi Benjamin cherche l'interruption de la logique mythique non pas dans un autre ordre politique, mais dans l'autre de la politique. Cette contribution examine néanmoins la possibilité d'une forme d'interruption du cercle mythique qui ne viendrait pas d'un au-delà de l'ordre de droit, ni ne l'exigerait, mais qui serait acceptée et préservée dans cet ordre même. Il s'agit d'une piste de réflexion ouverte par Benjamin lui-même - mais largement négligée par les nombreux commentateurs de son texte - dans l'excursus sur l'impunité originelle du mensonge, comme exemple d'une forme de communication non politisée dans l'ordre politique.

This contribution starts from the idea that it is not so much pre-modern political orders that appeal to mythical logic, but contemporary ones. Lacking an external authority, contemporary political orders cannot but erect themselves in an act of auto-constitution. With reference to Walter Benjamin's 'Critique of Violence', one could conceive of this act as the mythical encirclement of foundation and conservation. Although democratic regimes famously encourage ongoing debate about political affairs, this mythic circle seems to shut the door on fundamentally contesting the given order. This is why the interruption of the mythic logic is situated by Benjamin not in another political order, but in the other of politics. This article will however examine the possibility of interrupting the mythic circle not by opening or referring to a beyond of the legal order, but by making use of an originally accepted practice in this order. This possibility concerns a train of thought developed by Benjamin himself but largely neglected by the numerous commentators of his text : his digression on the impunity of the lie as an example of non-politicized form of communication within the political order.

\section{INDEX}

Keywords : Benjamin Walter, politics, myth, violence, representation

Mots-clés : Benjamin (Walter), politique, mythe, violence, représentation 


\section{AUTEUR}

\section{AUKJE VAN ROODEN}

Aukje van Rooden est professeur de philosophie à l'Université d'Amsterdam aux Pays-Bas. Elle a soutenu sa thèse de doctorat sur le mythe dans la politique. Ses publications concernent la philosophie politique, l'esthétique et la théorie littéraire. 